\title{
Aufgeholt, aber nicht aufgeschlossen: Wahrnehmungen und Wirkungen von TV-Duellen am Beispiel von Angela Merkel und Peer Steinbrïck 2013
}

\author{
Jürgen Maier, Thorsten Faas und Michaela Maier
}

Am 1. September 2013 erreichte der Bundestagswahlkampf seinen Höhepunkt: Drei Wochen vor der Wahl trafen sich Bundeskanzlerin Angela Merkel und ihr sozialdemokratischer Herausforderer, Peer Steinbrück, in Berlin-Adlershof zur einzigen Fernsehdebatte vor der Wahl. Fünf Fernsehsender - ARD, ZDF, RTL, ProSieben und Phoenix - strahlten das 93-minütige Duell aus, in dem die Kandidaten von vier Moderatoren - Anne Will, Maybrit Illner, Peter Klöppel und dem nicht unumstrittenen Entertainer Stefan Raab - unter Ausschluss von Publikum vor Ort befragt wurden. Insgesamt wurde die Debatte von 17,7 Millionen Zuschauern verfolgt. Dies entspricht einem Marktanteil von 50,6 Prozent. ${ }^{1}$ Diese Zahlen bestätigen wieder einmal den Befund zu früheren Bundestagswahlen, bei denen sich die Live-Diskussionen zwischen den Spitzenkandidaten als die bei weitem attraktivsten Politiksendungen im gesamten Wahlkampf herausstellten.

\section{Die Bedeutung von Fernsehdebatten und ihre Institutionalisierung in deutschen Wahlkämpfen}

Die Popularität des Formats Fernsehdebatte ergibt sich aus Sicht der Wähler vor allem aus der Möglichkeit, die Kandidaten und ihre Positionen zu den zentralen Wahlkampfthemen direkt miteinander vergleichen zu können. ${ }^{2}$ Dabei ist die aufzuwendende Zeit überschaubar. Zudem verspricht die Sendung aufgrund ihres kompetitiven Charakters einen gewissen Unterhaltungswert. Den Kandidaten eröffnet die Teilnahme an TV-Duellen die Chance, Millionen Wähler unter weitgehender Umgehung journalistischer Selektionskriterien direkt anzusprechen. Diese Vorzüge hat kein anderes Wahlkampfinstrument. Dass sich unter den Zuschauern auch viele politikferne Wähler befinden, die mit den traditionellen Mitteln des Wahlkampfs nur schwer zu erreichen sind, erhöht die Attraktivität von Fernsehdebatten zusätzlich, da diese nach den Erkenntnissen der Wahlkampfforschung besonders leicht zu beeinflussen sind. ${ }^{3}$ Allerdings bergen TV-Duelle auch Risiken. So gibt es zahlreiche, insbesondere US-amerikanische Beispiele, in denen Kandidaten mit ungünstigen Aussagen oder einem unangemessenen Auftreten ein erfolgreicheres Abschneiden verspielt haben und in der Folge als Verlierer dastanden. Oftmals sind solche Fehltritte so gravierend, dass sie in der

1 Vgl. Claudia Gscheidle / Heinz Gerhard, Berichterstattung zur Bundestagswahl 2013 aus Sicht der Zuschauer. Ergebnisse einer Repräsentativbefragung und des AGF-Fernsehpanels, in: Media Perspektiven 12/2013, S. 558 - 573, S. 565.

2 Vgl. Thorsten Faas / Jürgen Maier, Medienwahlkampf. Sind TV-Duelle nur Show und damit nutzlos?, in: Evelyn Bytzek / Sigrid Roßteutscher (Hrsg.), Der unbekannte Wähler? Mythen und Fakten über das Wahlverhalten der Deutschen, Frankfurt am Main 2011, S. 99 - 114.

3 Vgl. hierzu bereits Paul F. Lazarsfeld / Bernard Berelson / Hazel Gaudet, The People's Choice. How Voters Make Up Their Minds in a Presidential Campaign, New York 1944. 
verbleibenden Zeit bis zum Wahltag nicht mehr korrigiert werden können. ${ }^{4}$ Dafür sorgen auch Massenmedien: Mit ihrer umfassenden Vor- und Nachberichterstattung multiplizieren sie nicht nur die ohnehin große Reichweite von Fernsehdebatten. Sie reduzieren auch deren Inhalte und Verlauf, indem sie im Nachgang schlaglichtartig ganz bestimmte Aussagen oder Handlungen beleuchten - und eben gern auch Fehltritte von Kandidaten. ${ }^{5}$

In der Summe ergibt sich dennoch ein Bild, wonach Fernsehdebatten für alle beteiligten Akteure - Kandidaten, Medien, Wähler - unter dem Strich erhebliche Vorteile versprechen. TV-Duelle erzeugen somit eine „win-win-win“-Situation. ${ }^{6}$ Deshalb überrascht es nicht, dass solche Sendungen in vielen Demokratien ein fester Bestandteil von Wahlkämpfen sind. ${ }^{7}$ Dies gilt zwischenzeitlich auch für Deutschland: TV-Duelle nach US-amerikanischem Muster tauchten erstmals im Vorfeld der Hamburger Bürgerschaftswahl 1997 auf. ${ }^{8}$ Seit 2002 gibt es solche Fernsehdebatten auch im Rahmen von Bundestagswahlkämpfen. ${ }^{9}$ Insbesondere die Duelle zwischen den Kanzlerkandidaten haben die Akzeptanz dieses nicht unumstrittenen Formats deutlich erhöht. ${ }^{10}$ Während vor den Duellen zwischen Gerhard Schröder und Edmund Stoiber TV-Debatten nur vereinzelt in Landtagswahlkämpfen einen Platz gefunden hatten, trafen sich die Bewerber um das Amt des Regierungschefs in 15 von 16 Wahlen, die während der letzten Legislaturperiode des Deutschen Bundestages durchgeführt wurden, vor laufenden Kameras, um zentrale Wahlkampfthemen miteinander zu diskutieren (vgl. Abbildung 1). ${ }^{11}$ Zwischenzeitlich kann man also davon sprechen, dass solche Sendungen etabliert sind und Kandidaten ihnen nur mit großer Mühe und Ansehensverlusten aus dem Weg gehen können. ${ }^{12}$

4 Vgl. zum Beispiel Alan Schroeder, Presidential Debates. Fifty Years of High-Risk TV, New York 2008.

5 Vgl. zum Beispiel William L. Benoit / Kevin A. Stein / Glenn J. Hansen, Newspaper Coverage of Presidential Debates, in: Argumentation and Advocacy, 41. Jg. (2004), H. 1, S. 17 - 27.

6 Vgl. Jürgen Maier / Thorsten Faas, ,Miniature Campaigns' in Comparison. The German Televised Debates, 2002-09, in: German Politics, 20. Jg. (2011), H. 1, S. 75 - 91, S. 76.

7 Vgl. zum Beispiel die Übersicht in Pippa Norris, A Virtuous Circle. Political Communication in Postindustrial Societies, Cambridge 2000, S. 153.

8 Am 17. September 1997 traten der amtierende Bürgermeister Henning Voscherau (SPD) und sein Herausforderer Ole von Beust (CDU) vor die Kameras des NDR. Die 45-minütige Sendung wurde von etwa 500.000 Zuschauern verfolgt.

9 Vorläufer der heutigen TV-Duelle sind die sogenannten Elefantenrunden, zu denen die Spitzenpolitiker aller im Bundestag vertretenen Parteien (unter anderem auch die Kanzlerkandidaten der beiden Volksparteien) eingeladen wurden. Diese - mehrere Stunden in Anspruch nehmenden Sendungen fanden in der Regel drei Tage vor der Wahl statt und wurden im Vorfeld der Bundestagswahlen 1972 bis 1987 ausgestrahlt. Vgl. zum Beispiel Josef Klein, Elefantenrunden „Drei Tage vor der Wahl“. Die ARD-ZDF-Gemeinschaftssendung 1972-1987, Baden-Baden 1990.

10 In parlamentarischen Demokratien wurde und wird vereinzelt bemängelt, dass solche Sendungen nicht zur grundsätzlichen Architektur des politischen Systems passen. Hervorgehoben wird, dass TV-Duelle die Bedeutung von Personen herauf- und die Rolle von Parteien herunterspielen. Zudem wird kritisiert, dass in parlamentarischen Demokratien der politische Wettbewerb normalerweise zwischen mehr als zwei Parteien ausgetragen wird. Vgl. zum Beispiel Wolfgang Donsbach, Sechs Gründe gegen Fernsehduelle. Zur politischen Bewertung einer medialen Inszenierung, in: Die politische Meinung 396/2002, S. 19 - 25. Inzwischen ist diese Kritik aber zumindest in Deutschland weitgehend verstummt.

11 Bei den fünf Bundestags- und 34 Landtagswahlen zwischen 1997 und 2013 gab es insgesamt sechs beziehungsweise 38 TV-Duelle.

12 Vgl. Jürgen Maier / Thorsten Faas, a.a.O. (Fn. 6). 


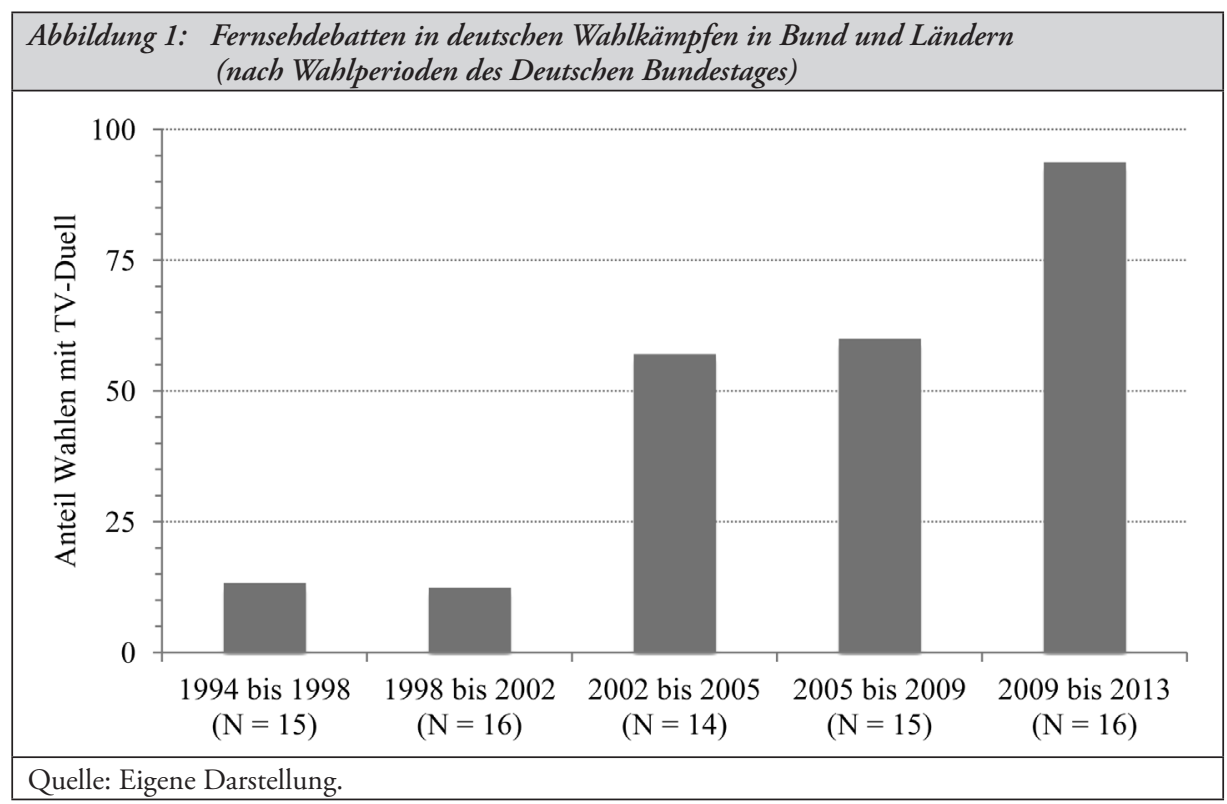

Angesichts der großen Strahlkraft von Fernsehdebatten stellt sich die Frage, welche Wirkungen diese wichtigsten Einzelereignisse in Wahlkämpfen haben. Dies gilt insbesondere für das TV-Duell 2013 zwischen Merkel und Steinbrück, denn die Hoffnungen gerade der SPD und ihres Spitzenkandidaten lagen auf diesem Großereignis. Tatsächlich konnte sich Steinbrück weitaus besser schlagen als erwartet: Merkel ging als klare Favoritin in die Debatte. ${ }^{13}$ Die an sie gerichteten Erwartungen konnte sie aber nicht erfüllen; in den meisten Umfragen wurde Steinbrück zum Sieger deklariert. ${ }^{14}$ Obwohl die Meinungsforschungsinstitute mit Blick auf die Wahlabsicht zu dem Ergebnis kamen, dass das TV-Duell keine große Wirkung hinterlassen hatte, legen andere Indikatoren - etwa Kandidatenbewertungen, Kompetenzeinschätzungen oder die Kanzlerpräferenz - nahe, dass es durchaus Einstellungen zu den beiden Kontrahenten beeinflusst hat. ${ }^{15}$ Allerdings haben die in den Medien präsentierten Zuschauerbefragungen aus einer Reihe von methodischen Gründen nur eine begrenzte Aussagekraft, wenn es um die Frage geht, welche Wirkungen von einem TV-

13 Vgl. zum Beispiel N24-Emnid-Umfrage zum TV-Duell, Mehrheit erwartet Sieg für Angela Merkel, 29. August 2013, http://www.presseportal.de/pm/13399/2545627/n24-emnid-umfragezum-tv-duell-mehrheit-erwartet-sieg-fuer-angela-merkel-aber-duell-ohne-grossen (Abruf am 24. Februar 2014).

14 Hiervon auszunehmen ist die im Auftrag des ZDF durchgeführte Umfrage der Forschungsgruppe Wahlen, die Merkel knapp als Siegerin gesehen hat; vgl. Forschungsgruppe Wahlen, TV-Duell 2013. Merkel gewinnt TV-Duell knapp, Steinbrück besser als erwartet, 1. September 2013, http://www.forschungsgruppe.de/Umfragen/Archiv_weitere_Umfragen/TV-Duell_2013/. Die Differenz zu anderen Umfragen ist vermutlich durch unterschiedliche Frageformate begründet; vgl. Jörg Schönenborn, ARD-DeutschlandTrend vom 5. September 2013, Duell-Umfragen: Unterschiede aufgeklärt, http://blog.tagesschau.de/2013/09/05/duell-umfragen-unterschiede-aufgeklaert/ (jeweils Abruf am 24. Februar 2014).

15 Vgl. Forschungsgruppe Wahlen, a.a.O. (Fn. 14). 
Duell ausgehen. Belastbare Ergebnisse zu den unmittelbaren Effekten von Fernsehdebatten müssen sich auf andere Daten stützen. Die nachfolgende Analyse untersucht deshalb Wahrnehmungen und ausgewählte Wirkungen der Fernsehdebatte 2013 auf die Zuschauer mit Hilfe von Experimentaldaten.

\section{Die Wirkung von Fernsehdebatten - ein (kurzer) Überblick über den Forschungsstand für Deutschland}

Die Debattenforschung hat zwischenzeitlich einen immensen Umfang erreicht. ${ }^{16}$ Die zahlreichen Analysen, die sich vorwiegend mit Fernsehduellen in den USA beschäftigen, konzentrieren sich auf drei große Forschungsfelder. Erstens untersuchen sie den Inhalt. Damit verbunden ist häufig die Frage, welche Strategien Kandidaten verfolgen, um möglichst gut in einer TV-Debatte abzuschneiden. Ein zweiter Forschungsstrang beschäftigt sich mit der Berichterstattung der Massenmedien. Typischerweise stehen dabei vor allem Umfang und Struktur der Anschlussberichterstattung im Mittelpunkt. Drittens wird der Blick auf die Wirkung von TV-Duellen gerichtet. Dieser Bereich ist mit Abstand am stärksten erforscht. Die vorliegenden Wirkungsstudien lassen sich danach einteilen, ob sie motivationale, kognitive, affektive oder verhaltensbezogene Effekte untersuchen. Des Weiteren kann man sie danach unterscheiden, ob sie direkten Wirkungen von Fernsehdebatten nachgehen oder aber sich für indirekte Effekte interessieren, die durch die - interpersonale oder massenmediale - Anschlusskommunikation entstehen. ${ }^{17}$

In jüngerer Vergangenheit ist ein weiterer, vierter Forschungsstrang hinzugekommen. Diese vorwiegend im Experimentallabor realisierten Untersuchungen thematisieren die Frage, wie TV-Debatten im Detail und in Echtzeit wahrgenommen und verarbeitet werden. Ein wichtiges Thema ist hier beispielsweise, welche Typen von Aussagen dazu beitragen, eine Debatte zu gewinnen oder wie Rezipienten zu ihrer Wahrnehmung eines Debattensiegers gelangen. Auf diese Weise versucht die Forschung, die zentralen psychologischen Mechanismen zu identifizieren, die bei der Verarbeitung von Debatteninhalten eine Rolle spielen.

In Deutschland hat sich die Debattenforschung seit den beiden Aufeinandertreffen von Schröder und Stoiber im Bundestagswahlkampf 2002 deutlich entwickelt. ${ }^{18}$ Seither sind

16 Einen Überblick über den Forschungsstand bieten zum Beispiel William L. Benoit / Glenn J. Hansen / Rebecca M. Verser, A Meta-Analysis of the Effects Viewing U.S. Presidential Debates, in: Communication Monographs, 70. Jg. (2003), H. 4, S. 335 - 350; Susan A. Hellweg / Michael Pfau I Steven R. Brydon, Televised Presidential Debates. Advocacy in Contemporary America, New York 1992; Kathleen Hall Jamieson / David S. Birdsell, Presidential Debates. The Challenge of Creating an Informed Electorate, Oxford 1988; Elihu Katz / Jacob J. Feldman, The Debates in the Light of Research. A Survey of Surveys, in: Sidney Kraus (Hrsg.), The Great Debates. Kennedy vs. Nixon, Bloomington 1962, S. 173 - 223; Lanoue, David L. I Peter R. Schrott, The Joint Press Conference. The History, Impact, and Prospects of American Presidential Debates, New York 1991; David O. Sears / Steven H. Chaffee, Uses and Effects of the 1976 Debates. An Overview of Empirical Studies, in: Sidney Kraus (Hrsg.), The Great Debates. Carter vs. Ford. Bloomington 1979, S. 223 - 261; Sidney Kraus, Televised Presidential Debates and Public Policy, Mahwah 2000.

17 Allerdings sind viele der vorliegenden Wirkungsstudien aufgrund methodischer Probleme nicht in der Lage, zwischen direkten und indirekten Effekten zu differenzieren.

18 Für eine Analyse der bis dahin ausgestrahlten Elefantenrunden vgl. zum Beispiel Peter R. Schrott, Wahlkampfdebatten im Fernsehen von 1972 bis 1987. Politikerstrategien und Wählerreaktion, 
Dutzende Untersuchungen zu den TV-Duellen 2002, 2005 und 2009 auf Bundesebene vorgelegt worden. ${ }^{19}$ Deren Ergebnisse erschöpfend zusammenzufassen, würde an dieser Stelle den Rahmen sprengen und den zum Teil sehr differenzierten Analysen nicht gerecht werden. Stattdessen sollen im Folgenden nur sehr knapp zentrale Ergebnisse deutscher Wirkungsstudien umrissen werden.

Ähnlich wie für US-amerikanische Debatten kann auch für die Bundesrepublik gezeigt werden, dass die Rezeption von TV-Duellen grundsätzlich zum Auf- und Ausbau von politischem Wissen führt. ${ }^{20}$ In besonderem Maße können dabei bildungsferne Wählersegmente neue Kenntnisse erwerben. ${ }^{21}$ Damit gelingt es diesen Bevölkerungsgruppen, die bestehende Wissenskluft zu ressourcenstärkeren Wählern ein Stück weit zu schließen. Grundsätzlich unterscheiden sich die Wissenszuwächse zwischen den in einem TV-Duell angesprochenen Themen deutlich. Lernerfolge sind dann besonders wahrscheinlich, wenn sich die Kandidaten einfach ausdrücken und Informationen wiederholt werden. ${ }^{22}$

Während die US-Forschung mehrheitlich davon ausgeht, dass Fernsehdebatten politische Einstellungen eher verstärken als verändern können, zeigt sich für Deutschland ein etwas anderes Bild. Viele Studien belegen, dass sich die Bewertung aller an einer Fernsehdebatte teilnehmenden Politiker verbessert - und zwar häufig über die Grenzen von Parteilagern hinweg. ${ }^{23}$ Grundsätzlich profitieren also alle Kandidaten von einem TV-Duell. Die

in: Max Kaase / Hans-Dieter Klingemann (Hrsg.), Wahlen und Wähler. Analysen aus Anlaß der Bundestagswahl 1987, Opladen 1990, S. 647 - 674.

19 Nur für zwei TV-Duelle auf Landesebene liegen bisher Analysen vor: erstens für die Fernsehdebatte 2006 in Mecklenburg-Vorpommern; vgl. Marcus Maurer, Sagen Bilder mehr als tausend Worte? Die Relevanz verbaler und visueller Information für die Urteilsbildung über Personen im Fernsehen, in: Medien \& Kommunikationswissenschaft, 57. Jg. (2009), H. 2, S. 198 - 216; zweitens für das TV-Duell 2011 in Baden-Württemberg; vgl. zum Beispiel die Beiträge in Marko Bachl / Frank Brettschneider / Simon Ottler (Hrsg.), Das TV-Duell in Baden-Württemberg 2011, Wiesbaden 2013.

20 Vgl. Ursula Dehm, Fernsehduelle im Urteil der Zuschauer. Eine Befragung des ZDF zu einem neuen Sendungsformat bei der Bundestagswahl 2002, in: Media Perspektiven 12/2002, S. 600 - 609; dies., Das TV-Duell 2005 aus Zuschauersicht. Eine Befragung des ZDF zum Wahlduell zwischen Herausforderin Angela Merkel und Kanzler Gerhard Schröder, in: Media Perspektiven 12/2005, S. 627 - 637; dies., Das TV-Duell 2009 aus Zuschauersicht. Dreistufige Befragung zum Wahlduell zwischen Kanzlerin Angela Merkel und Herausforderer Frank-Walter Steinmeier, in: Media Perspektiven 12/2009, S. 651 - 661; Thorsten Faas / Jürgen Maier, a.a.O. (Fn. 2); Jürgen Maier, Eine Basis für rationale Wahlentscheidungen? Die Wirkungen des TV-Duells auf politische Kenntnisse, in: Marcus Maurer / Carsten Reinemann / Jürgen Maier / Michaela Maier, Schröder gegen Merkel. Wahrnehmung und Wirkung des TV-Duells 2005 im Ost-West-Vergleich, Wiesbaden 2007, S. 129 - 143; Marcus Maurer / Carsten Reinemann, Schröder gegen Stoiber. Nutzung, Wahrnehmung und Wirkung der TV-Duelle, Wiesbaden 2003; dies., Learning versus Knowing. Effects of Misinformation in Televised Debates, in: Communication Research, 33. Jg. (2006), H. 6, S. $489-506$.

21 Vgl. Thorsten Faas / Jürgen Maier, a.a.O. (Fn. 2); Jürgen Maier, a.a.O. (Fn. 20).

22 Vgl. Jürgen Maier, a.a.O. (Fn. 20).

23 Vgl. zum Beispiel Marko Bachl, Die Wirkung des TV-Duells auf die Bewertung der Kandidaten und die Wahlabsicht, in: ders. / Frank Brettschneider / Simon Ottler (Hrsg), a.a.O. (Fn. 19), S. 171 - 198; Christian Holst / Rüdiger Schmidt-Beck / Thorsten Faas, Kampagnendynamik! Der Nutzen von Rolling-Cross-Section Designs für die Kommunikationsforschung mit einem Beispiel aus der Wahlforschung, in: planung \& analyse 5/2006, S. 54 - 58; Jürgen Maier / Thorsten Fads, The Affected German Voter. Televized Debates, Follow-Up Communication and Candidate Evaluation, in: Communications, 28. Jg. (2003), S. 383 - 404; Marcus Maurer / Carsten Reinemann, Schröder gegen Stoiber, a.a.O. (Fn. 20), S. 157 f. 
Höhe des Ansehensgewinns fällt allerdings nicht für jeden Kandidaten und auch nicht für jede Dimension, entlang der Politiker bewertet werden können, identisch aus. ${ }^{24}$ Experimental- und Panelstudien haben immer wieder gezeigt, dass bis zu einem Drittel der Rezipienten ihre Kanzlerpräferenz aufgrund der TV-Debatte ändern. ${ }^{25}$ Besonders starke Wirkungen haben Fernsehduelle auf Zuschauer ohne Parteiidentifikation. Allerdings sind auch bei Parteianhängern Wanderungsbewegungen zu erkennen. ${ }^{26}$ Umstritten ist, ob Fernsehdebatten einen Beitrag zur Personalisierung des Wahlverhaltens leisten, das heißt dass Wähler nach der Rezeption eines TV-Duells eher unpolitische Merkmale zur Beurteilung der Kandidaten heranziehen und Kandidatenbewertungen größeres Gewicht für ihre Wahlentscheidung beimessen. ${ }^{27}$

Im Gegensatz zur bislang stark erforschten Wirkung von TV-Duellen auf Kandidatenbewertungen ist die Frage nach der Bedeutung von Fernsehdebatten für die Wahrnehmung und Beurteilung von Sachfragen kaum untersucht. Dies ist durchaus überraschend, denn die Kandidaten verwenden den überwiegenden Anteil ihrer Aussagen darauf, über Inhalte - und nicht etwa über Personen - zu sprechen. Die wenigen für die Bundesrepublik vorliegenden Befunde deuten darauf hin, dass von TV-Duellen keine bedeutsamen Agenda-Setting-Effekte ausgehen. ${ }^{28}$ Dagegen können sich die Wahrnehmungen von Problemlösungskompetenzen sehr wohl verschieben: Fast ein Drittel der Teilnehmer einer Untersuchung zur Merkel-Steinmeier-Debatte 2009 haben ihre Kompetenzzuschreibung nach der Sendung modifiziert. $^{29}$

Im Hinblick auf Wahlbeteiligung und Wahlverhalten stimuliert die Rezeption von TVDuellen die Partizipationsbereitschaft. ${ }^{30}$ Die gemessenen Zuwächse sind dabei durchaus

24 Vgl. zum Beispiel Frank Brettschneider / Marko Bachl, Kandidaten-Images und ihre Bedeutung für die Wahlabsicht, in: dies. I Simon Ottler (Hrsg.), a.a.O. (Fn. 19), S. 199 - 217; Jürgen Hofrichter, Die Rolle der TV-Duelle im Bundestagswahlkampf 2002, in: Frank Brettschneider I Jan van Deth / Edeltraut Roller (Hrsg.), Die Bundestagswahl 2002. Analysen der Wahlergebnisse und des Wahlkampfes, Wiesbaden 2004, S. 51 - 73; Jürgen Maier / Thorsten Faas, a.a.O. (Fn. 23); Marcus Maurer / Carsten Reinemann, Schröder gegen Stoiber, a.a.O. (Fn. 20), S. $158 \mathrm{ff}$.

25 Vgl. Jürgen Maier, Erfolgreiche Überzeugungsarbeit. Urteile über den Debattensieger und die Veränderung der Kanzlerpräferenz, in: Marcus Maurer / Carsten Reinemann / Jürgen Maier / Michaela Maier, a.a.O. (Fn. 20), S. 91 - 109; Jürgen Maier / Thorsten Faas, Das TV-Duell 2009 - langweilig, wirkungslos, nutzlos? Ergebnisse eines Experiments zur Wirkung der Fernsehdebatte zwischen Angela Merkel und Frank-Walter Steinmeier, in: Heinrich Oberreuter (Hrsg.), Am Ende der Gewissheiten. Wähler, Parteien und Koalitionen in Bewegung, München 2011, S. 147 - 166; vgl. auch Jürgen Hofrichter, a.a.O. (Fn. 24).

26 Vgl. Jürgen Maier / Thorsten Faas, a.a.O. (Fn. 25).

27 Vgl. zum Beispiel Marcus Maurer / Carsten Reinemann, a.a.O. (Fn. 20); dies., Personalisierung durch Priming. Die Wirkungen des TV-Duells auf die Urteilskriterien der Wähler, in: Marcus Maurer / Carsten Reinemann / Jürgen Maier / Michaela Maier, a.a.O. (Fn. 20), S. 111 - 128.

28 Vgl. Jürgen Hofrichter, a.a.O. (Fn. 24); Jürgen Maier/ Thorsten Faas, a.a.O. (Fn. 25).

29 Vgl. Jürgen Maier / Thorsten Faas, a.a.O. (Fn. 25).

30 Vgl. zum Beispiel Thorsten Faas / Jürgen Maier, Chancellor-Candidates in the 2002 Televised Debates, in: German Politics, 13. Jg. (2004), H. 2, S. 300 - 316; Markus Klein, Der Einfluss der beiden TV-Duelle im Vorfeld der Bundestagswahl 2002 auf die Wahlbeteiligung und die Wahlentscheidung. Eine log-lineare Pfadanalyse auf der Grundlage von Paneldaten, in: Zeitschrift für Soziologie, 34 Jg. (2005), H. 3, S. 207 - 222; Jürgen Maier / Thorsten Faas, Schröder gegen Stoiber. Wahrnehmung, Verarbeitung und Wirkung der Fernsehdebatten im Bundestagswahlkampf 2002, in: Jürgen W. Falter / Oscar W. Gabriel / Bernhard Weßels (Hrsg.), Wahlen und Wäh- 
bemerkenswert und auch nach Kontrolle von Drittvariablen deutlich messbar. ${ }^{31}$ Dabei werden vor allem politisch wenig Interessierte durch TV-Duelle mobilisiert. ${ }^{32}$

Auch das letzte Element der Wirkungskette, die Wahlabsicht, wird beeinflusst - zumindest in Deutschland. Im Unterschied zu Befunden aus den USA haben Fernsehdebatten hierzulande einen zum Teil massiven Einfluss auf die Parteiwahl der Rezipienten; dabei wird dieser Effekt durch die Wahrnehmung des Debattensiegers moderiert. ${ }^{33}$ Die gemessenen Zusammenhänge deuten einerseits darauf hin, dass Zuschauer bei einem Sieg des „eigenen “ Kandidaten in ihrer Absicht bestärkt werden, ihre Stimme für ihn beziehungsweise seine Partei abzugeben. ${ }^{34}$ Andererseits ist zu erkennen, dass sie von ihrer ursprünglichen Wahlabsicht abrücken, wenn sie eine Niederlage des eigenen Kandidaten konstatieren (müssen). ${ }^{35}$ Massiv sind die Effekte von Fernsehdebatten bei der - stetig größer werdenden - Gruppe der parteipolitisch ungebundenen Wähler. Wird ein Kandidat als Debattensieger gesehen, steigt die Wahrscheinlichkeit einer Stimmabgabe zugunsten seiner Partei im Mittel um 30 bis 40 Prozentpunkte. ${ }^{36}$

Alles in allem sind TV-Duelle damit nicht nur wegen ihrer hohen Reichweite, sondern auch aufgrund ihrer offenkundig massiven Wirkungen wichtige Meilensteine in heutigen Wahlkämpfen. Sie scheinen eine echte Hilfe zu sein, wenn es für Bürger darum geht, eine Wahlentscheidung zu treffen.

ler. Analysen aus Anlass der Bundestagswahl 2002, Wiesbaden 2005, S. 77 - 101; dies., a.a.O. (Fn. 6); Jürgen Maier / Thorsten Faas / Michaela Maier, Mobilisierung durch Fernsehdebatten. Zum Einfluss des TV-Duells auf die politische Involvierung und die Partizipationsbereitschaft, in: Bernhard Weßels / Harald Schoen / Oscar W. Gabriel (Hrsg.), Wahlen und Wähler. Analysen aus Anlass der Bundestagswahl 2009, Wiesbaden 2013, S. 79 - 96.

31 Vgl. Jürgen Maier / Thorsten Faas, a.a.O. (Fn. 6), S. 82.

32 Vgl. zum Beispiel Thorsten Faas / Jürgen Maier, a.a.O. (Fn. 30); Jürgen Maier / Thorsten Faas, a.a.O. (Fn. 6); dies., a.a.O. (Fn. 30).

33 Vgl. zum Beispiel Thorsten Faas / Jürgen Maier, a.a.O. (Fn. 30); Markus Klein, a.a.O. (Fn. 30); ders., Die TV-Duelle. Events ohne Effekt?, in: Manfred Güllner / Hermann Dülmer / Markus Klein / Dieter Ohr / Markus Quandt / Ulrich Rosar / Hans-Dieter Klingemann, Die Bundestagswahl 2002. Eine Untersuchung im Zeichen hoher politischer Dynamik, Wiesbaden 2005, S. 143 - 159; Markus Klein / Manuela Pötschke, Haben die beiden TV-Duelle im Vorfeld der Bundestagswahl 2002 den Wahlausgang beeinflusst? Eine Mehrebenenanalyse auf der Grundlage eines 11-Wellen-Kurzfristpanels, in: Jürgen W. Falter / Oscar W. Gabriel / Bernhard Weßels (Hrsg.), a.a.O. (Fn. 30), S. 357 - 370; Markus Klein / Ulrich Rosar, Wirkungen des TV-Duells im Vorfeld der Bundestagswahl 2005 auf die Wahlentscheidung. Eine empirische Analyse unter besonderer Berücksichtigung von Medieneinflüssen auf die Siegerwahrnehmung und subjektive Erwartungshaltungen an die Debattenperformanz der Kandidaten, in: Kölner Zeitschrift für Soziologie und Sozialpsychologie, 59. Jg. (2007), H. 1, S. 81 - 104; Jürgen Maier, Wahlkampfkommunikation und Wahlverhalten, in: Hans Rattinger / Oscar W. Gabriel / Jürgen W. Falter (Hrsg.), Der gesamtdeutsche Wähler. Stabilität und Wandel des Wählerverhaltens im wiedervereinigten Deutschland, Baden-Baden 2007, S. 385 - 411; Jürgen Maier / Thorsten Faas, a.a.O. (Fn. 6); dies., a.a.O. (Fn. 25); dies., a.a.O. (Fn. 30); Michaela Maier, Verstärkung, Mobilisierung, Konversion - möglich ist alles! Die Wirkungen des TV-Duells auf die Wahlabsicht, in: Marcus Maurer / Carsten Reinemann / Jürgen Maier / Michaela Maier, a.a.O. (Fn. 20), S. 145 - 165.

34 Vgl. zum Beispiel Markus Klein, a.a.O. (Fn. 30); Jürgen Maier / Thorsten Faas, a.a.O. (Fn. 6).

35 Vgl. zum Beispiel Marko Bachl, Die Wirkungen des TV-Duells auf die Bewertung der Kandidaten und die Wahlabsicht, in: ders. / Frank Brettschneider / Simon Ottler (Hrsg.), a.a.O. (Fn. 19), S. 173 - 198; Jürgen Maier / Thorsten Fads, a.a.O. (Fn. 6).

36 Vgl. Jürgen Maier / Thorsten Faas, a.a.O. (Fn. 6), S. 87. 
Diese zahlreichen Nachweise von Effekten, die im unmittelbaren Nachgang zu TV-Duellen zu beobachten sind, werfen (mindestens) zwei Anschlussfragen auf, nämlich erstens: Was genau an den TV-Debatten ist für diese Effekte verantwortlich, wie funktionieren die Mechanismen, an deren Ende sie stehen? Und zweitens: Wie stabil und dauerhaft sind diese Effekte?

Während die Zahl der Wirkungsstudien bemerkenswert hoch ist, ist der Forschungsstand zu diesen beiden Fragen bescheidener. Mit Blick auf die Stabilität von Effekten aber unterstreichen die vorliegenden Studien sehr wohl die Wichtigkeit dieser Forschungsfrage. Einflüsse auf Kandidateneinstellungen etwa sind eher fragiler Natur. Dabei kommt gerade der Anschlusskommunikation über ein TV-Duell besondere Bedeutung zu; sie kann dafür sorgen, dass Imagegewinne und Personalisierungstendenzen nach nur wenigen Tagen wieder verschwunden sind. ${ }^{37}$ Auch potenzielle Verschiebungen von Themenkompetenzen erweisen sich mitunter als instabil. ${ }^{38}$ Selbst Wahrnehmungen der Zuschauer zur Sinnhaftigkeit und Nützlichkeit eines Duells werden durch die mediale Anschlusskommunikation beeinflusst. ${ }^{39}$

Die vorliegenden Studien zur Wahrnehmung von TV-Duellen zeigen, dass gerade diese über Richtung und Stärke verhaltensrelevanter Debatteneffekte entscheidet. Damit rückt unmittelbar die Frage nach den entscheidenden Determinanten für diese Wahrnehmung in den Fokus. Die naheliegende Vermutung, dass sich Fernsehzuschauer angesichts der Fülle an dargebotenen Informationen an einfachen Heuristiken - etwa der Parteibindung oder den eigenen Erwartungen an den Ausgang eines Duells - orientieren, trifft dabei nur zum Teil zu. Während Erwartungen keine besondere Bedeutung zukommt ${ }^{40}$, prägt eine vorhandene Parteiidentifikation tatsächlich die Wahrnehmung der Leistung eines Kontrahenten im Verlauf der Debatte, die wiederum dem letztlichen Urteil über den Debattensieger vorgelagert ist. ${ }^{41} \mathrm{Im}$ Unterschied zu anderen Wahlkampfinformationen fällt es Wählern bei

37 Vgl. zum Beispiel Jürgen Hofrichter, a.a.O. (Fn. 24); Christian Holst / Rüdiger Schmidt-Beck / Thorsten Faas, a.a.O. (Fn. 23); Jürgen Maier / Michaela Maier, Das TV-Duell 2005. Katalysator für die Personalisierung des Wahlverhaltens?, in: Frank Brettschneider / Oskar Niedermayer / Bernhard Weßels (Hrsg.), Die Bundestagswahl 2005. Analysen des Wahlkampfes und der Wahlergebnisse, Wiesbaden 2007, S. 219 - 232; Harald Schoen, Der Kanzler, zwei Sommerthemen und ein Foto-Finish. Priming-Effekte bei der Bundestagswahl 2002, in: Frank Brettschneider / Jan van Deth / Edeltraud Roller (Hrsg.), Die Bundestagswahl 2002. Analysen der Wahlergebnisse und des Wahlkampfes, Wiesbaden 2004, S. 23 - 50; Jürgen Maier / Thorsten Faas, Debates, Media, and Social Networks. How Interpersonal and Mass Communication Affected the Evaluation of the Televised Debates in the 2002 German Election, in: Angela Schorr / Stefan Seltmann (Hrsg.), Changing Media Markets in Europe and Abroad. New Ways of Handling Information and Entertainment Content, New York 2006, S. 43 - 62.

38 Vgl. Harald Schoen, a.a.O. (Fn. 37).

39 Vgl. Thorsten Faas / Jürgen Maier, a.a.O. (Fn. 2).

40 Vgl. Marko Bachl, a.a.O. (Fn. 23); Jürgen Maier, a.a.O. (Fn. 25); Jürgen Maier / Thorsten Fads, Debattenwahrnehmung und Kandidatenorientierung. Eine Analyse von Real-Time-Responseund Paneldaten zu den Fernsehduellen im Bundestagswahlkampf 2002, in: Zeitschrift für Medienpsychologie, 16. Jg. (2004), H. 1, S. 26 - 35; Michaela Maier / Jesper Strömbäck, Advantages and Limitations of Comparing Audience Responses to Televised Debates. A Comparative Study of Germany and Sweden, in: Jürgen Maier / Michaela Maier / Marcus Maurer / Carsten Reinemann / Vincent Meyer (Hrsg.), Real-Time Response Measurement in the Social Sciences, Frankfurt am Main 2009, S. 97 - 116.

41 Vgl. zum Beispiel Marko Bachl, a.a.O. (Fn. 23); Jürgen Maier, a.a.O. (Fn. 25); Jürgen Maier I Marcus Maurer / Carsten Reinemann / Thorsten Faas, Reliability and Validity of Real-Time Re- 
TV-Debatten aber vergleichsweise schwer, die Kandidaten dauerhaft durch die parteipolitische Brille zu betrachten. Damit eröffnen sich den Kandidaten Möglichkeiten, nicht nur im eigenen, sondern auch im gegnerischen Lager zu punkten. ${ }^{42}$

Wie dies gelingen kann, wird kontrovers diskutiert. Untersuchungen zur Wahrnehmung von bestimmten Typen von Aussagen legen nahe, dass Stellungnahmen, denen nahezu jeder zustimmen kann, oder - weniger eindeutig - vage gehaltene Aussagen lagerübergreifend Zustimmung finden. ${ }^{43}$ Die Wirkung von Angriffen auf den politischen Gegner ist demgegenüber komplexer: Während einige Studien eine generelle Polarisierung des Publikums entlang parteipolitischer Linien beobachten ${ }^{44}$, finden andere Untersuchungen weniger eindeutige Effekte. ${ }^{45}$ Insgesamt scheint die Wirkung von Angriffen stark von externen Rahmenbedingungen abzuhängen. ${ }^{46}$

Aufbauend auf diesem Überblick wird im Folgenden das Duell 2013 zwischen Angela Merkel und Peer Steinbrück betrachtet und eingeordnet. Zunächst geht es um die (sekundengenaue) Wahrnehmung des Ereignisses durch die Zuschauer: Womit konnten Merkel und Steinbrück punkten? An welchen Stellen mussten sie Federn lassen? Darauf aufbauend

sponse Measurement. A Comparison of Two Studies of a Televised Debate in Germany, in: International Journal of Public Opinion Research, 19. Jg. (2007), H. 1, S. 53 - 73; Michaela Maier I Jesper Strömbäck, a.a.O. (Fn. 40); Carsten Reinemann / Jürgen Maier / Thorsten Faas / Marcus Maurer, Reliabilität und Validität von RTR-Messungen. Ein Vergleich zweier Studien zur zweiten Fernsehdebatte im Bundestagswahlkampf 2002, in: Publizistik, 50. Jg. (2005), H. 1, S. 56 - 73.

42 Vgl. Thorsten Faas / Jürgen Maier, Mobilisierung, Verstärkung, Konversion? Ergebnisse eines Experiments zur Wahrnehmung der Fernsehduelle im Vorfeld der Bundestagswahl 2002, in: PVS, 45. Jg. (2004), H. 1, S. $55-72$.

43 Vgl. Marko Bachl, Die Wahrnehmungen des TV-Duells, in: ders. / Frank Brettschneider / Simon Ottler (Hrsg.), a.a.O. (Fn. 19), S. 135 - 169; Marcus Mauer / Carsten Reinemann, a.a.O. (Fn. 20); dies., Schröder gegen Merkel. Eine Analyse der Zuschauereindrücke während des TV-Duells, in: Heinrich Oberreuter (Hrsg.), Unentschieden. Die erzwungene Koalition, München 2009, S. 119 - 140; Frederike Nagel, Die Wirkung verbaler und nonverbaler Kommunikation in TV-Duellen. Eine Untersuchung am Beispiel von Gerhard Schröder und Angela Merkel, Wiesbaden 2012; Carsten Reinemann / Marcus Maurer, Unifying or Polarizing? Short-Term Effects and Post-Debate Consequences of Different Rhetorical Strategies in Televised Debates, in: Journal of Communication, 55. Jg. (2005), H. 4, S. 775 - 794; dies, Populistisch und unkonkret. Die unmittelbare Wahrnehmung des TV-Duells, in: dies. / Jürgen Maier / Michaela Maier, a.a.O. (Fn. 20), S. $53-89$.

44 Vgl. Marcus Mauer / Carsten Reinemann, a.a.O. (Fn. 20); dies., Schröder gegen Merkel, a.a.O. (Fn. 43); dies., Unifying or Polarizing?, a.a.O. (Fn. 43); dies., Populistisch und unkonkret, a.a.O. (Fn. 43).

45 Vgl. Marko Bachl, a.a.O. (Fn. 43); Jürgen Maier, „Frau Merkel wird doch noch Kritik ertragen können...". Inhalt, Struktur, Wahrnehmung und Wirkung des wirtschaftspolitischen Teils der Fernsehdebatte 2005, in: Oscar W. Gabriel / Bernhard Weßels / Jürgen W. Falter (Hrsg.), Wahlen und Wähler. Analysen aus Anlass der Bundestagswahl 2005, Wiesbaden 2009, S. 177 - 201; Arne Spieker, Licht ins Dunkel der TV-Duelle. Rhetorische Strategien und ihre Wirkungen im TV-Duell 2009. Eine empirische Analyse mittels Real-Time-Response Measurement, in: Josef Ferdinand Haschke / André Michael Moser (Hrsg.), Politik - Deutsch, Deutsch - Politik. Aktuelle Trends und Fachergebnisse, Berlin 2011, S. 75 - 93.

46 Dazu gehört gerade auch die Rolle nonverbaler Kommunikation, die - wie vorliegende Studien zeigen - auch einen Einfluss haben kann: Frederike Nagel, a.a.O. (Fn. 43); dies. / Marcus Maurer I Carsten Reinemann, Is There a Visual Dominance in Political Communication? How Verbal, Visual, and Vocal Communication Shape Viewers' Impressions of Political Candidates, in: Journal of Communication, 62. Jg. (2012), H. 5, S. $833-850$. 
wird gefragt, wer von beiden aus Sicht der Zuschauer als Sieger aus der Debatte hervorging und welche Faktoren dafür entscheidend waren. Drittens soll ermittelt werden, ob sich wie die vorliegende Forschung nahelegt - die Debattenleistungen Merkels und Steinbrücks auch in den kandidatenbezogenen Einstellungen der Zuschauer niederschlagen - und für wie lange.

\section{Datenbasis}

Grundlage für die nachfolgenden Analysen ist ein Modul der German Longitudinal Election Study (GLES). ${ }^{47}$ Konkret kommen vor allem Daten zum Einsatz, die auf der Messung von Debatteneffekten im Experimentallabor basieren. Der Vorteil von Experimentaldaten gegenüber klassischen Umfragen liegt darin, dass Rezipienten die TV-Debatte unter vollständig kontrollierten Bedingungen verfolgen. Gemessene Veränderungen sind deshalb zuverlässig auf die Rezeption des Duells - und nicht auf andere, nicht kontrollierbare Einflüsse - zurückzuführen. Die hier verwendeten Daten basieren auf insgesamt 247 Probanden, die in Räumen der Universitäten Koblenz-Landau und Mainz am Abend des TV-Duells direkt vor und direkt nach der Sendung ausführlich zu ihren politischen Orientierungen und Verhaltensabsichten befragt wurden. ${ }^{48} 208$ Personen verfolgten das Duell live in Räumlichkeiten der beiden Universitäten mit Bild und Ton, 39 konnten die Debatte nur hören. 205 Personen hatten darüber hinaus die Gelegenheit, in Echtzeit und sekundengenau ihre unmittelbaren Eindrücke von den Kandidaten mit Hilfe spezieller Eingabetechniken - einer so genannten Real-Time-Response-Messung - zu dokumentieren. ${ }^{49}$ Diese Daten liegen auf Sekundenbasis vor und ermöglichen einen Einblick in die Wahrnehmung der Aussagen und des Auftretens der beiden Kandidaten. Um die Stabilität möglicher Debatteneffekte zu prüfen, gleichzeitig aber auch mögliche Wirkungen von Anschlusskommunikation zu berücksichtigen, wurden die Teilnehmer einige Tage nach dem Ereignis nochmals befragt. ${ }^{50}$

47 Die Daten der GLES sind unter http://www.gesis.org/wahlen/gles/ öffentlich zugänglich (Abruf am 18. Dezember 2013).

48 Die Personen wurden auf der Basis eines Quotierungsplans rekrutiert, der eine Gleichverteilung der Merkmale Geschlecht, Alter (18 bis 39 Jahre vs. 40 Jahre und älter), Bildung (Haupt- und Realschule vs. Fachabitur beziehungsweise Abitur) und Parteiidentifikation (CDU/CSU, FDP vs. SPD, Bündnis 90/Die Grünen, Die Linke vs. keine Parteiidentifikation) vorsah. Es hat sich als schwierig erwiesen, formal gering gebildete Wähler sowie Anhänger der Regierungsparteien beziehungsweise parteipolitisch ungebundene Wähler für die Teilnahme an der Untersuchung zu gewinnen. Für die nachfolgenden Auswertungen wurde deshalb eine Gewichtung nach Parteiidentifikation vorgenommen, so dass die drei ins Auge gefassten Wählergruppen - erstens Anhänger von CDU/CSU und FDP, zweitens Anhänger von SPD, Bündnis 90/Die Grünen und der Linken, drittens Wähler ohne Parteiidentifikation beziehungsweise mit einer Identifikation für eine andere Partei - mit gleichem Gewicht in die Analysen eingehen.

4988 Personen wurden mit Drehreglern, 117 Probanden mit Druckknöpfen ausgestattet. Ein Vergleich der beiden Real-Time-Response-Systeme findet sich zum Beispiel in Carsten Reinemann I Jürgen Maier / Thorsten Faas / Marcus Maurer, a.a.O. (Fn. 40).

50 Eine vierte und letzte Befragung, die nach dem Tag der Bundestagswahl stattfand, hat an dieser Stelle jedoch keine Relevanz. 


\section{Ergebnisse}

\subsection{Zur unmittelbaren Wahrnehmung des TV-Duells}

Abbildung 2 zeigt die „Fieberkurve“ des Duells 2013, also die (durchschnittliche) Bewertung seines Verlaufs. An welchen Stellen und mit welchen Aussagen konnten Merkel und Steinbrück punkten? Ein erstes Ausrufezeichen setzte die Kanzlerin, als sie nach der Frage, ob sie Peer Steinbrück aufgrund der Schwierigkeiten im SPD-Wahlkampf bemitleiden würde, betonte, darum würde es nicht gehen, sondern es würde vielmehr die Frage zur Debatte stehen, wem man eher zutraue, die Probleme Deutschlands zu lösen (vgl. Markierung 1 in Abbildung 2). Auch wenn sie in der Folgezeit weitere Aussagen traf, die von den Rezipienten deutlich positiv bewertet wurden - den Umgang mit Griechenland (2), ihre Ansätze zur Lösung der Euro-Krise (3), die Höhe des Lohnniveaus (4), das Konzept der Mindestrente (5), die Kritik an den SPD-Plänen, Pensionen zu besteuern (6), die Absage an ein militärisches Eingreifen Deutschlands in Syrien (7) und ihre positive Bilanz der Arbeit der Großen Koalition in den Jahren 2005 bis 2009 (8) - blieb die Bewertung dieses ersten Highlights aus der Startphase des Duells (mit einem Zustimmungswert von 5,12 auf einer Skala von 1 bis 7) unübertroffen.

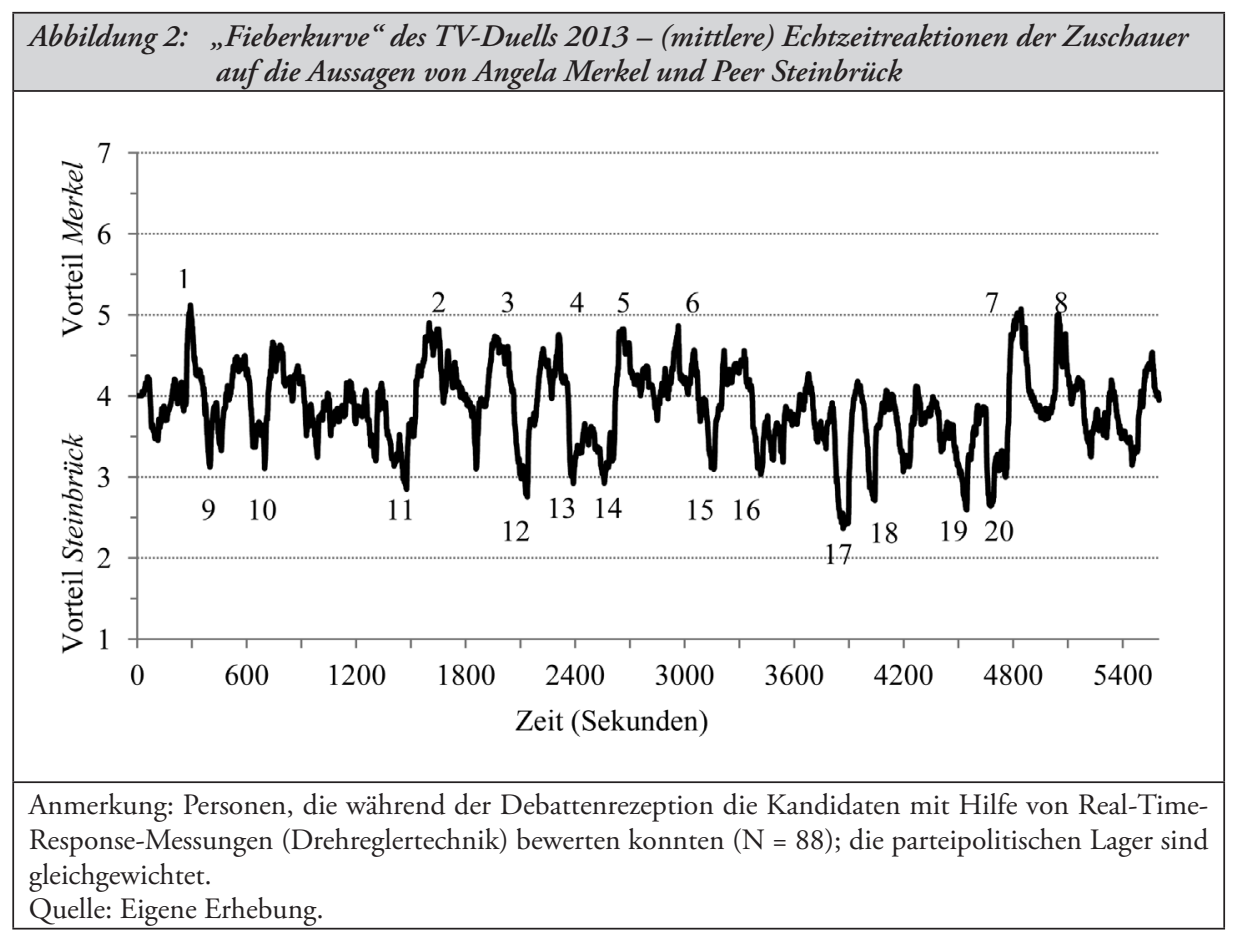

Im Vergleich zu Merkel gelang es Steinbrück häufiger und oftmals ausgeprägter, die Zuschauer zu überzeugen. Erstmals punkten konnte er mit der Aufzählung von Problemen, die aus seiner Sicht von der amtierenden Regierung nicht gelöst worden seien (9), gefolgt von seinem Plädoyer für einen flächendeckenden Mindestlohn (10). Auch als er Solidarität 
mit Griechenland forderte (11), einen härteren Umgang mit im Ausland angelegten Unternehmensgewinnen empfahl (12) und die Zunahme von gering vergüteten Arbeitsverhältnissen anprangerte (13), folgten ihm die Zuschauer. Ebenfalls auf starke Zustimmung stießen seine Positionen zu den Themen Rente $(14,15)$, Pflege $(17)^{51}$ und Kinderbetreuung (18). Punkten konnte er auch beim Thema Energiewende (16) sowie mit seinen Einschätzungen zum Umgang mit den USA in der NSA-Affäre (19) und der Absage an eine militärische Lösung des Syrien-Konflikts (20). Insgesamt legen diese unmittelbaren Zuschauereindrücke nahe, dass der Herausforderer sich über den Verlauf der Debatte hinweg besser „verkaufen“ konnte als die Amtsinhaberin.

\subsection{Zur Wahrnehmung des Ausgangs der Debatte}

Die sekundengenauen Wahrnehmungen finden auch in der Gesamtbewertung der Debatte ihren Niederschlag, denn die unmittelbare Nachbefragung der Teilnehmer an der Experimentalstudie zeigt, dass Steinbrück das TV-Duell gewonnen hat (vgl. Tabelle 1). Dabei konnte er die an ihn gerichteten Erwartungen deutlich übertreffen, denn direkt vor der Debatte hatte noch eine Mehrheit der Befragten erwartet, dass die Kanzlerin das Duell für sich entscheiden würde.

Allerdings wurde Steinbrück nur im eigenen Lager und unter den parteiungebundenen Wählern als Sieger gesehen. Im christlich-liberalen Lager war auch nach dem Duell eine überwältigende Mehrheit der Auffassung, die Kanzlerin habe gewonnen oder mindestens genauso gut abgeschnitten wie ihr Herausforderer. ${ }^{52}$ Wie unsere Befragung einige Tage nach dem Duell zeigt (Ergebnisse nicht tabellarisch ausgewiesen), sind diese Wahrnehmungen der Debattenleistung sehr stabil.

\begin{tabular}{|l|c|c|c|c|c|}
\hline Tabelle 1: Erwarteter und wahrgenommener Debattensieger \\
\hline & $\begin{array}{c}\text { erwarteter } \\
\text { Sieger } \\
\text { direkt vor } \\
\text { der Debatte }\end{array}$ & \multicolumn{5}{|c|}{ wahrgenommener Sieger direkt nach der Debatte } \\
\cline { 3 - 6 } & 42 & 29 & $\begin{array}{c}\text { Parteiidenti- } \\
\text { fikation: CDU/ } \\
\text { CSU, FDP }\end{array}$ & $\begin{array}{c}\text { Parteiidenti- } \\
\text { fikation: SPD, } \\
\text { Grüne, Linke }\end{array}$ & $\begin{array}{c}\text { keine / andere } \\
\text { Parteiidenti- } \\
\text { fikation }\end{array}$ \\
\hline Merkel (in \%) & 19 & 38 & 9 & 16 & 21 \\
Steinbrück (in \%) & 39 & 33 & 42 & 64 & 42 \\
unentschieden (in \%) & 230 & 230 & 55 & 113 & 37 \\
\hline N (ungewichtet) & \multicolumn{5}{|l}{} \\
\hline Quelle: Eigene Berechnung.
\end{tabular}

Wie bedeutsam die Aussagen und das Auftreten der Kandidaten für das Siegerurteil der Zuschauer ist, zeigt die in Abbildung 3 dargestellte Analyse. Wen Fernsehzuschauer nach der TV-Debatte als Gewinner wahrgenommen haben, hängt zu großen Teilen von der per

51 Hier erreichte Steinbrück seine beste Bewertung $(2,36)$.

52 Auch wenn die hier vorgestellten Experimentaldaten nicht repräsentativ sind, zeichnen sie doch ein ähnliches Bild wie einige der nach dem TV-Duell veröffentlichten Umfragen. 
Real-Time-Response-Messung erfassten Bewertung der Debattenleistung ab. Einen deutlich geringeren Einfluss auf das Siegerurteil hat die vor dem Duell geäußerte Erwartung, wer gewinnen wird. Ohne direkte Wirkung ist die Parteibindung. Diese schlägt sich aber - etwa so stark wie die individuellen Erwartungen - auf die Bewertung der Debattenleistung nieder. Außerdem beeinflusst die Parteibindung die vor dem Duell geäußerte Einschätzung, wer die Diskussion als Sieger beenden wird, färbt also die dem Siegerurteil vorgelagerten Einstellungen. Allerdings ist ihr Einfluss nicht so stark, dass für die Zuschauer schon vorab feststeht, wer in ihren Augen der Sieger sein wird. Diese Einschätzung hängt in erster Linie von der Performanz der Kandidaten ab. Für die Kandidaten besteht also ein großer Spielraum, durch einen gelungenen Auftritt die Zuschauer von sich zu überzeugen.

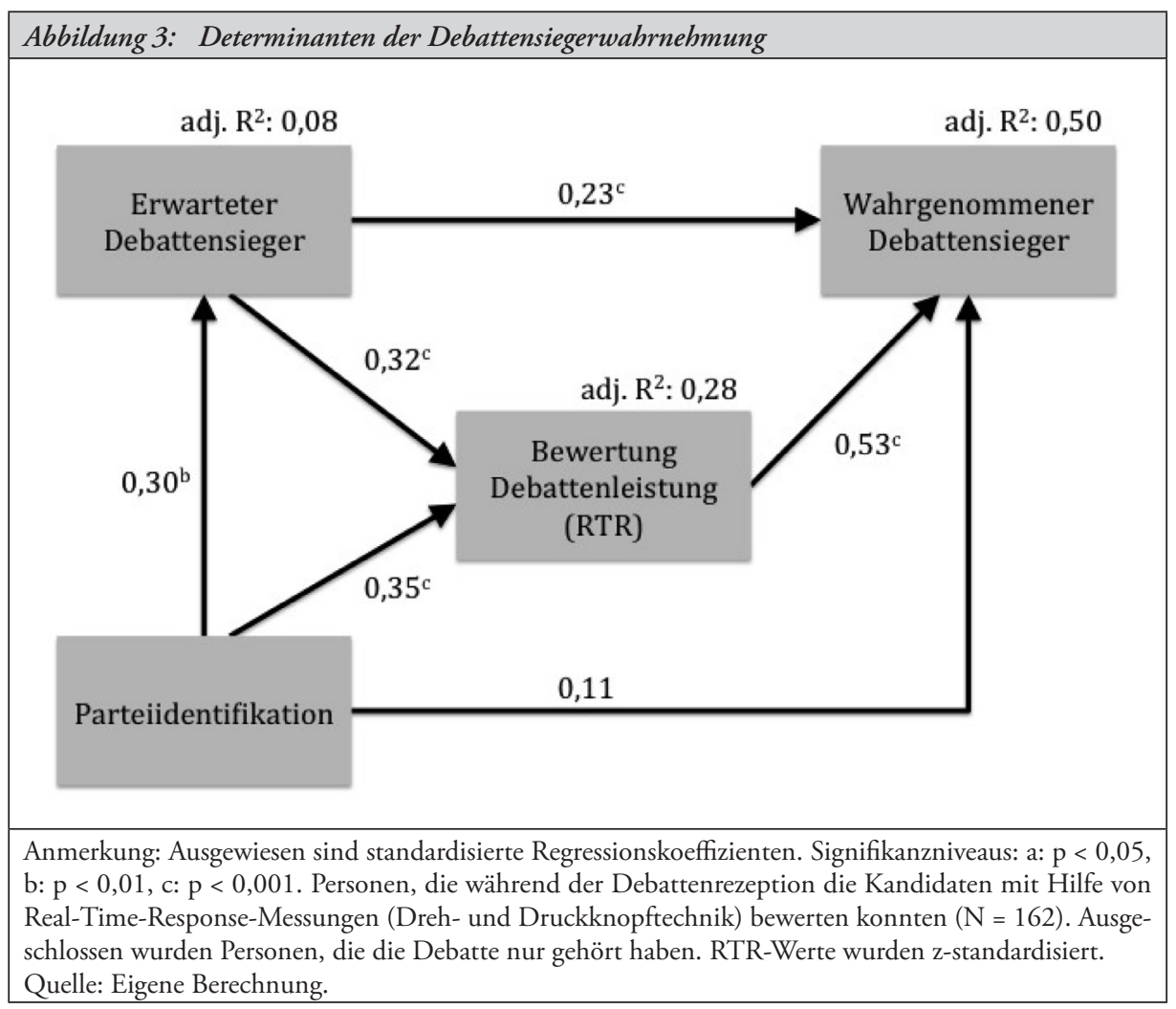

\subsection{Zur Wirkung des TV-Duells auf die Kandidatenbewertungen}

Aus der Sicht der Kandidaten ist die relevante Frage, ob es in einer TV-Debatte gelingen kann, auf sie bezogene Einstellungen und letztlich das Wahlverhalten der Rezipienten zu ändern. Das Fernsehduell 2013 hat, bezogen auf den Aspekt der Kandidatenbewertung, sehr unterschiedliche Wirkungen für die Kanzlerin und ihren Herausforderer entfaltet (vgl. Tabelle 2). Während sich die Gesamtbewertung von Angela Merkel verschlechterte, konnte Peer Steinbrück deutlich zulegen. Der Imageverlust der Kanzlerin ist dabei vor allem bei 
parteipolitisch ungebundenen Wählern deutlich. Innerhalb von 90 Minuten hat sich ihr Ansehen hier um fast 0,7 Skalenpunkte verringert. Demgegenüber verbuchte Steinbrück in allen Wählergruppen einen kräftigen Imagegewinn von mindestens 0,9 Skalenpunkten.

\begin{tabular}{|c|c|c|c|c|}
\hline \multicolumn{5}{|c|}{$\begin{array}{l}\text { Tabelle 2: Unmittelbarer Einfluss der TV-Debatte auf die Veränderung von Einstellungen zu den } \\
\text { Kanzlerkandidaten }\end{array}$} \\
\hline & Gesamt & $\begin{array}{l}\text { Parteiidentifikation: } \\
\text { CDU/CSU, FDP }\end{array}$ & $\begin{array}{l}\text { Parteiidentifikation: } \\
\text { SPD, Grüne, Linke }\end{array}$ & $\begin{array}{l}\text { keine / andere Partei- } \\
\text { identifikation }\end{array}$ \\
\hline \multicolumn{5}{|l|}{ Merkel } \\
\hline Gesamtbewertung & $-0,24^{a}$ & 0,06 & $-0,13$ & $-0,69^{\mathrm{b}}$ \\
\hline Kompetenz & 0,04 & $0,16^{a}$ & $-0,06$ & 0,03 \\
\hline Führungsstärke & $0,11^{\mathrm{a}}$ & $0,26^{c}$ & 0,00 & 0,06 \\
\hline Integrität & $-0,01$ & 0,07 & $-0,11$ & 0,02 \\
\hline $\begin{array}{l}\text { Persönliche } \\
\text { Sympathie }\end{array}$ & $0,13^{\mathrm{b}}$ & $0,13^{\mathrm{a}}$ & 0,14 & 0,13 \\
\hline \multicolumn{5}{|l|}{ Steinbrück } \\
\hline Gesamtbewertung & $1,04^{\mathrm{c}}$ & $0,91^{\mathrm{c}}$ & $1,23^{\mathrm{c}}$ & $0,96^{\mathrm{c}}$ \\
\hline Kompetenz & $0,21^{\mathrm{c}}$ & 0,11 & $0,24^{b}$ & $0,29^{c}$ \\
\hline Führungsstärke & $0,28^{\mathrm{c}}$ & $0,22^{\mathrm{b}}$ & $0,28^{\mathrm{b}}$ & $0,36^{c}$ \\
\hline Integrität & $0,31^{\mathrm{c}}$ & $0,28^{c}$ & $0,30^{\mathrm{c}}$ & $0,35^{\mathrm{c}}$ \\
\hline $\begin{array}{l}\text { Persönliche } \\
\text { Sympathie }\end{array}$ & $0,41^{\mathrm{c}}$ & $0,23^{\mathrm{b}}$ & $0,44^{\mathrm{c}}$ & $0,57^{c}$ \\
\hline $\mathrm{N}$ (ungewichtet) & Min. 214 & Min. 53 & Min. 111 & Min. 50 \\
\hline \multicolumn{5}{|c|}{$\begin{array}{l}\text { Anmerkung: Ausgewiesen sind Veränderungen der Kandidatenbewertungen zwischen der Befragung di- } \\
\text { rekt vor und direkt nach der TV-Debatte. Positive Werte indizieren eine Verbesserung, negative Werte } \\
\text { eine Verschlechterung der Bewertung. Die Gesamtbewertung wurde auf einer } 11 \text {-Punkte-Skala gemes- } \\
\text { sen. Alle anderen Dimensionen sind aus jeweils zwei Fragen zusammengesetzte Skalen, die sich im Wer- } \\
\text { tebereich } 1 \text { bis } 5 \text { bewegen. Signifikanzniveaus: a: } p<0,05 \text {, b: } p<0,01 \text {, c: } p<0,001 \text {. } \\
\text { Quelle: Eigene Berechnung. }\end{array}$} \\
\hline
\end{tabular}

Mit Blick auf Einzelaspekte der Kandidatenbewertung zeigt sich für Merkel hier ein etwas positiveres Bild. So wurde sie nach dem Duell als signifikant führungsstärker und sympathischer wahrgenommen. Diese Zugewinne gehen aber einzig auf die Verbesserung ihres Ansehens in den eigenen Reihen zurück. Demgegenüber konnte sie weder im Lager des politischen Gegners noch bei den unabhängigen Wählern Boden gut machen. Steinbrück verbesserte sein Ansehen hingegen entlang aller Dimensionen. Am stärksten wandelte sich dabei der Eindruck, den die Wähler von seiner Persönlichkeit hatten. Imagegewinne sind in allen Wählersegmenten zu beobachten. Die stärkste Wirkung konnte Steinbrück bei den parteipolitisch ungebundenen Wählern erzeugen.

Trotz seiner im Vergleich zu Merkel deutlichen Imagegewinne konnte er den - hier nicht tabellarisch ausgewiesenen - Vorsprung der Kanzlerin zwar verringern, aber nicht mit ihr gleichziehen. ${ }^{53}$ Dieser Befund bestätigt sich auch mit Blick auf die Kanzlerpräferenz. Wäh-

53 Die einzige Ausnahme ist die wahrgenommene Führungsstärke. Hier lag Steinbrück vor dem Duell gleichauf mit Merkel. Durch die TV-Debatte konnte er sich einen Vorsprung erarbeiten. 
rend sich vor der Debatte 50 Prozent der Probanden im Falle einer Direktwahl für Merkel und 21 Prozent für Steinbrück entschieden hätten, kam die Kanzlerin nach dem Duell auf 48, ihr Herausforderer auf 29 Prozent. Mit anderen Worten: Der Vorsprung der Amtsinhaberin reduzierte sich von 29 auf 19 Prozentpunkte (vgl. Tabelle 3). Diese Entwicklung ist in allen Wählergruppen zu beobachten.

\begin{tabular}{|c|c|c|c|c|}
\hline \multicolumn{5}{|c|}{$\begin{array}{l}\text { Tabelle 3: Vorsprung von Angela Merkel auf Steinbrück bei der Kanzlerpräferenz vor und nach } \\
\text { dem TV-Duell }\end{array}$} \\
\hline & Gesamt & $\begin{array}{l}\text { Parteiidentifikation: } \\
\text { CDU/CSU, FDP }\end{array}$ & $\begin{array}{l}\text { Parteiidentifikation: } \\
\text { SPD, Grüne, Linke }\end{array}$ & $\begin{array}{l}\text { keine / andere } \\
\text { Parteiidentifikation }\end{array}$ \\
\hline $\begin{array}{l}\text { direkt vor dem } \\
\text { Duell (in \%) }\end{array}$ & 29 & 86 & -22 & 26 \\
\hline $\begin{array}{l}\text { direkt nach dem } \\
\text { Duell (in \%) }\end{array}$ & 19 & 78 & -39 & 16 \\
\hline $\mathrm{N}$ (ungewichtet) & 230 & 55 & 113 & 62 \\
\hline \multicolumn{5}{|c|}{$\begin{array}{l}\text { Anmerkung: Ausgewiesen ist der Saldo zwischen der Präferenz für Merkel und Steinbrück in Prozent- } \\
\text { punkten. Positive Werte indizieren einen Vorsprung für Merkel, negative Werte einen Vorsprung für } \\
\text { Steinbrück. } \\
\text { Quelle: Eigene Berechnung. }\end{array}$} \\
\hline
\end{tabular}

\subsection{Zur Stabilität der Wirkung des TV-Duells auf die Kandidatenbewertung}

Wie oben bereits dargestellt, haben sich die Wahrnehmungen, dass Steinbrück das Duell 2013 für sich entscheiden konnte, im Nachgang zur Debatte verfestigt. Das heißt aber keineswegs, dass die positiven Effekte auf sein Image auch dauerhafter Natur sind; schließlich geht der Wahlkampf nach dem Duell weiter, und andere Faktoren jenseits des Duells nehmen Einfluss auf das Bild der Kandidaten.

Tatsächlich prägen negative Koeffizienten das Bild, das Tabelle 4 widerspiegelt. Inhaltlich bedeutet dies: Das Ansehen beider Kontrahenten verschlechterte sich einige Tage nach dem Duell wieder gegenüber der Messung unmittelbar nach dem Duell. Einige der beobachtbaren Rückgänge sind dabei auch in ihrer Größenordnung beachtlich; dies gilt insbesondere für die wahrgenommene Führungsstärke beider Kandidaten; bei Steinbrück betrifft es auch die Wahrnehmung seiner Integrität und persönlichen Sympathie. Gleichwohl bleibt festzuhalten, dass diese negativen Veränderungen seinen Ansehensgewinn durch die Fernsehdebatte keineswegs aufzehrten. Vielmehr war sein Bild auch wenige Tage nach dem Duell noch positiver als vor dem Duell. Dies zeigt sich auch in der Kanzlerfrage. Obwohl Steinbrück einen kleinen Teil seines Zugewinns wieder einbüßte, gilt auch hier: Der Abstand ist wenige Tage nach dem Duell kleiner, als er vor dem Duell war - selbst wenn die Kanzlerin noch immer deutlich vor ihrem Herausforderer liegt. ${ }^{54}$

54 Der Vorsprung Merkels gegenüber Steinbrück in der „K-Frage“ betrug wenige Tage nach dem Duell 80 Prozentpunkte in den eigenen Reihen und 21 Prozentpunkte bei den parteipolitisch unabhängigen Wählern. Demgegenüber wuchs Steinbrücks Rückhalt im eigenen Lager weiter an; sein Vorsprung auf die Kanzlerin vergrößerte sich auf 42 Prozentpunkte. Betrachtet man alle Wähler, lag Merkel unverändert 19 Prozentpunkte vor ihrem Herausforderer. 


\begin{tabular}{|c|c|c|c|c|}
\hline & Gesamt & $\begin{array}{l}\text { Parteiidentifikation: } \\
\text { CDU/CSU, FDP }\end{array}$ & $\begin{array}{l}\text { Parteiidentifikation: } \\
\text { SPD, Grüne, Linke }\end{array}$ & $\begin{array}{c}\text { keine / andere } \\
\text { Parteiidentifikation }\end{array}$ \\
\hline \multicolumn{5}{|l|}{ Merkel } \\
\hline Gesamtbewertung & $-0,17$ & $-0,34^{b}$ & $-0,23$ & 0,05 \\
\hline Kompetenz & $-0,05$ & $-0,01$ & $-0,06$ & $-0,08$ \\
\hline Führungsstärke & $-0,13^{b}$ & $-0,13^{\mathrm{a}}$ & $-0,06$ & $-0,21^{\mathrm{a}}$ \\
\hline Integrität & $-0,05$ & $-0,04$ & $-0,03$ & $-0,08$ \\
\hline $\begin{array}{l}\text { Persönliche } \\
\text { Sympathie }\end{array}$ & $-0,06$ & $-0,08$ & $-0,06$ & $-0,03$ \\
\hline \multicolumn{5}{|l|}{ Steinbrück } \\
\hline Gesamtbewertung & $-0,08$ & $-0,23$ & $-0,14$ & 0,14 \\
\hline Kompetenz & $-0,06$ & $-0,02$ & $-0,05$ & $-0,10$ \\
\hline Führungsstärke & $-0,10^{\mathrm{a}}$ & $-0,08$ & $-0,08$ & $-0,14^{\mathrm{a}}$ \\
\hline Integrität & $-0,16^{c}$ & $-0,24^{\mathrm{b}}$ & $-0,12$ & $-0,14$ \\
\hline $\begin{array}{l}\text { Persönliche } \\
\text { Sympathie }\end{array}$ & $-0,13^{\mathrm{b}}$ & $-0,08$ & $-0,12$ & $-0,20^{\mathrm{b}}$ \\
\hline $\mathrm{N}$ (ungewichtet) & Min. 219 & Min. 53 & Min. 108 & Min. 58 \\
\hline \multicolumn{5}{|c|}{$\begin{array}{l}\text { Anmerkung: Ausgewiesen sind Veränderungen der Kandidatenbewertungen zwischen der Befragung di- } \\
\text { rekt nach und wenige Tage nach der TV-Debatte. Positive Werte indizieren eine Verbesserung, negative } \\
\text { Werte eine Verschlechterung der Bewertung. Die Gesamtbewertung wurde auf einer } 11 \text {-Punkte-Skala } \\
\text { gemessen. Alle anderen Dimensionen sind aus jeweils zwei Fragen zusammengesetzte Skalen, die sich im } \\
\text { Wertebereich } 1 \text { bis } 5 \text { bewegen. Signifikanzniveaus: a: } \mathrm{p}<0,05 \text {, b: } \mathrm{p}<0,01 \text {, c: } \mathrm{p}<0,001 \text {. } \\
\text { Quelle: Eigene Berechnung. }\end{array}$} \\
\hline
\end{tabular}

\section{TV-Duelle: wirkungsvolles Wablkampfinstrument mit wachsender Bedeutung}

Auch bei der Bundestagswahl 2013 war die TV-Debatte zwischen den Kanzlerkandidaten der beiden Volksparteien der Höhepunkt des Wahlkampfs: Wie schon bei den Kanzlerduellen der vorangegangenen Wahlen erzeugte dieses Format die mit Abstand größte Zuschauerresonanz, die für eine politische Sendung im Wahlkampf gemessen wurde. Erneut wurde das Aufeinandertreffen von Kanzlerin und ihrem Herausforderer umfassend medial vorund nachbereitet. Und auch die Kandidaten nahmen das TV-Duell ernst und versuchten, damit auf den Wahlausgang Einfluss zu nehmen. Während das Ziel von Merkel darin bestand, ihren Vorsprung gegenüber Steinbrück zu verteidigen und der schwarz-gelben Koalition weitere vier Jahre Regierungszeit zu sichern, galt es für den SPD-Spitzenkandidaten, seine vermeintlich „letzte Chance" noch einmal spannend zu machen.

55 Vgl. zum Beispiel Letzte Chance für Steinbrück, in: Frankfurter Rundschau Online vom 30. August 2013, http://www.fr-online.de/bundestagswahl---hintergrund/tv-duell-letzte-chance-fuersteinbrueck,23998104,24165924.html (Abruf am 13. Januar 2014). 
Paradoxerweise haben beide ihr Ziel erreicht. Zweifelsohne holte Steinbrück durch die Fernsehdebatte 2013 auf. Er konnte nicht nur entgegen aller Erwartungen das Duell für sich entscheiden, sondern es gelang ihm auch, seine Gesamtbewertung deutlich zu verbessern. Zudem schätzen ihn die Zuschauer entlang verschiedener für die Wahrnehmung von Politikern relevanter Teildimensionen nach dem Duell deutlich besser ein als vorher. Außerdem konnte er seinen Abstand in der „K-Frage“ verringern. Alle hier angelegten Indikatoren zeigen also, dass Steinbrücks Auftritt gelungen war. Auch wenn er seinen Höhenflug nicht konservieren konnte, waren die durch die Fernsehdebatte ausgelösten Effekte kein Strohfeuer. Zwar zeigen Daten, die wenige Tage nach dem Duell erhoben wurden, dass sich die Bewertungen des Herausforderers wieder verschlechterten - allerdings bleibt insgesamt das Ergebnis bestehen, dass Steinbrück mit dem TV-Duell den Abstand zur Kanzlerin reduzieren konnte. Möglicherweise hat seine Debattenperformanz also mit dazu beigetragen, der SPD den Weg zurück an die Macht zu ebnen.

Aber auch Angela Merkel konnte letztlich zufrieden sein, denn obwohl ihr Herausforderer aufholte, war er letztlich nicht in der Lage, zu ihr aufzuschließen. Der Vorsprung, mit dem die Kanzlerin in das TV-Duell ging, war groß genug, um Steinbrück auf Distanz zu halten. Merkel hat zudem bewiesen, dass sie die Konsequenzen eines TV-Duells richtig eingeschätzt hatte, als sie wie schon in den beiden vorangegangenen Bundestagswahlkämpfen darauf beharrte, sich nur für eine Fernsehdebatte zur Verfügung zu stellen. Es gelang ihr auch, Steinbrück in den Tagen bis zur Wahl auf Abstand zu halten - nicht zuletzt deshalb, weil der deutliche Trend, den das TV-Duell zu seinen Gunsten ausgelöst hatte, sich in den darauffolgenden Tagen nicht fortsetzte. Am Ende verbuchte die Union sogar kräftige Zugewinne und stand dicht davor, die absolute Mehrheit der Bundestagsmandate zu erringen.

Die Bundestagswahl 2013 hat erneut deutlich gemacht, wie wichtig Fernsehdebatten sind. Sie sind nicht nur ein wesentlicher Fixpunkt des Wahlkampfs, sondern verfügen auch über das Potenzial, die politischen Einstellungen - und damit prinzipiell auch das Wahlverhalten - der Fernsehzuschauer zu beeinflussen. Da aus wahlsoziologischer Sicht mit dem Bedeutungsverlust von Parteibindungen auch die Zahl derjenigen Wähler zunimmt, die für Wahlkampfbotschaften empfänglich sind, dürfte auch die Relevanz von TV-Duellen weiter wachsen. Entscheidend dürfte dabei auch sein, wann diese im Wahlkampf stattfinden, denn die Effekte von Duellen sind, wie die hier präsentierten Ergebnisse, keineswegs unveränderlich. ${ }^{56} 2013$ hat die Kanzlerin das Duell bewusst drei Wochen vor den Wahltag - und damit früher als bei vorangegangenen Wahlen - legen lassen. Man darf gespannt sein, worauf sich die Kandidaten 2017 einigen werden. Dass es zu mindestens einem TV-Duell kommen wird, dürfte schon heute klar sein.

56 Zum Zusammenhang von Stärke und Stabilität von Debatteneffekten und dem Timing von TVDuellen vgl. Jürgen Maier / Thorsten Faas, a.a.O. (Fn. 23). 